\title{
Perception of Midwives Towards Magnesium Sulfate Use at Chatinkha Maternity Wing in Blantyre, Malawi: A Qualitative Study
}

This article was published in the following Dove Press journal: International Journal of Women's Health

\author{
Maria Chifuniro Chikalipo ${ }^{1}{ }^{\prime}$ \\ Lester Kapanda Phiri $\mathbb{D D}^{2}$ \\ Neggie Mndolo (iD ${ }^{3}$ \\ Christina Ruth Mbiza ${ }^{4}$ \\ Patricia Khisi ${ }^{4}$ \\ Eunice Golombe (iD ${ }^{4}$ \\ Phylos Bonongwe ${ }^{4}$ \\ Ellen Mbweza Chirwa' \\ Alfred Maluwa (iD) ${ }^{5}$
}

'University of Malawi, Kamuzu College of Nursing, Blantyre, Malawi; ${ }^{2}$ University of Malawi College of Medicine, Blantyre, Malawi; ${ }^{3}$ Malawi College of Health Sciences, Blantyre, Malawi; ${ }^{4}$ Ministry of Health, Queen Elizabeth Central Hospital, Blantyre, Malawi; ${ }^{5}$ Malawi University of Science and Technology, Blantyre, Malawi

Correspondence: Maria Chifuniro

Chikalipo

Kamuzu College of Nursing

Email mchikalipo@kcn.unima.mw
Introduction: Globally, magnesium sulfate $\left(\mathrm{MgSO}_{4}\right)$ has been recognized as the drug of choice for preventing and controlling fits among women with severe pre-eclampsia and eclampsia, respectively. Improper use of magnesium sulfate has been reported globally. Therefore, actionable findings for improving magnesium sulfate use are needed. This study aims at understanding the views of midwives towards $\mathrm{MgSO}_{4}$ use to inform an intervention whose objective is to improve $\mathrm{MgSO}_{4}$ use among the midwives.

Methods: An exploratory qualitative study was conducted from July to September 2018. We conducted 10 in-depth interviews and a focus group discussion with midwives. All the interviews were audio taped and transcribed verbatim. Data were managed by NVivo version 10.0 and analyzed thematically.

Results: We identified one overarching theme: "Inadequate governing approaches on management of clients on $\mathrm{MgSO}_{4}$ " with corresponding subthemes; in adequate preparation on magnesium sulfate administration; inconsistent formula, regimen and guidelines/protocols on magnesium sulfate use and lack of resources.

Conclusion: Midwives perceived $\mathrm{MgSO}_{4}$ use as a demanding activity due to inadequate training, inconsistent tools, and lack of resources. While periodic in-service training should be intensified to improve $\mathrm{MgSO}_{4}$ use, necessary resources should be provided, such as blood pressure machines, more midwives, and protocols.

Keywords: magnesium sulfate, Malawi, midwives, perception and resources

\section{Introduction}

Pre-eclampsia and eclampsia continue to cause morbidity and mortality among childbearing women globally. Preeclampsia and eclampsia contribute to $14 \%$ of maternal deaths worldwide, and the majority of these deaths occur in Low and Medium Income Countries (LMICs), Malawi inclusive, with some variations among countries. ${ }^{1,7}$ For instance, between 2016 and 2017, out of 10,000 deliveries, rates of eclampsia ranged from 19.6/10,000 in Zambia to 142.0/10,000 in Sierra Leone and 107.1/10,000 in Malawi. ${ }^{7}$ On the other hand maternal deaths due to eclampsia ranged from $1.1 \%$ in Zambia to $15.5 \%$ in Sierra Leone, with $3.2 \%$ in Malawi. ${ }^{7}$ Additionally, pre-eclampsia and eclampsia is the third leading cause of maternal deaths in Malawi. ${ }^{8,10}$ Currently the maternal mortality ratio (MMR) is at 439 per 100,000 live births according to 2015 data reported in a Malawi Demographic Health Survey of 2017 while, according to UNICEF and the WHO, the 2015 MMR is 634 per 100,000 live births. ${ }^{11}$ Although there are variations in the 
estimated maternal mortality ratios, MMR in Malawi is high, more than the expected 140 per 100,000 live births according to Sustainable Development Goal targets. Therefore, interventions to prevent eclampsia from occurring are urgently needed to improve maternal outcomes.

Clinical evidence acknowledges magnesium sulfate $\left(\mathrm{MgSO}_{4}\right)$ as the drug of choice globally for severe preeclampsia and eclampsia. ${ }^{612,15}$ Additionally the Magpie study and other related studies have demonstrated that $\mathrm{MgSO}_{4}$ use in reducing the risk of eclampsia is rarely associated with serious side-effects, deeming it a safe drug. ${ }^{6,16,17}$ Additionally the WHO has included $\mathrm{MgSO}_{4}$ under the World Health Organization Essential Medicines List and United Nations 13 life-saving commodities list, 2011. ${ }^{15}$ Despite $\mathrm{MgSO}_{4}$ being a reliable drug for controlling and preventing convulsions among women diagnosed with severe pre-eclampsia and eclampsia, ${ }^{1}$ its use including management of clients on $\mathrm{MgSO}_{4}$ is unsatisfactory. ${ }^{18,20}$ Fear of magnesium sulfate toxicity and the perception that magnesium sulfate has to be administered in intensive care are some of the reported factors associated with improper use of $\mathrm{MgSO}_{4}$ among healthcare workers. ${ }^{2,4,19}$ Therefore improving $\mathrm{MgSO}_{4}$ use and its management would require understanding perceptions towards $\mathrm{MgSO}_{4}$ use among the midwives. Such information is scant in Malawi. This study sought to answer the following question "What are the views of midwives towards magnesium sulfate use among women with severe pre-eclampsia and eclampsia?". The aim is to assess the perception of midwives towards magnesium sulfate use and its management to inform an intervention whose objective is to improve $\mathrm{MgSO}_{4}$ use.

\section{Conceptual Framework}

Consolidated framework for implementation research (CFIR) guided analysis of the data and discussion of the results. The framework consists of five main domains, which are; intervention, inner and outer setting, individual characteristics, and process by which the implementation is accomplished. ${ }^{21}$ In our study, the intervention is magnesium sulfate use, which is defined as "administration of magnesium sulfate to pregnant women diagnosed with severe pre-eclampsia or eclampsia and their monitoring to determine progress". On the other hand inner and outer settings include; the needs of clients on magnesium sulfate and the availability of resources, while individual characteristics involve the individual's beliefs in their own capabilities to implement an action within a specific context. Finally, the process which involves engaging appropriate individuals to carry out an action and ensuring that the action is executed according to a set standard to ensure quality. ${ }^{22}$ The process also involves proper planning, monitoring and evaluation and provision of support. The framework was chosen as it provides defined constructs that can assist in improving practice by assessing potential drivers and barriers to inform implementation of a practice in a better way in a specific context. ${ }^{23}$

\section{Methods}

\section{Study Design}

We conducted an exploratory cross-sectional qualitative study from July to September 2018 among midwives to explore and describe their perceptions towards magnesium sulfate use and its management among women with severe pre-eclampsia and eclampsia. The design provided the opportunity to have a broader understanding of the subject matter. ${ }^{24,25}$ In addition, we employed focus group discussion (FGD) and in-depth interviews (IDIs). Collecting data through a FGD and IDIs allowed gathering of rich contextualized data and understanding of the phenomena as the data collection methods complimented each other. $^{26,27}$

\section{Study Setting}

The study was conducted in the maternity wing (Chatinkha) of Queen Elizabeth Central Hospital $(\mathrm{QECH})$. The hospital is located in Blantyre, an industrial city. It is one of the largest public hospitals in Malawi and functions both as a secondary facility for Blantyre district and a tertiary facility for all the three regions of Malawi. It serves a population of about 1 million, including the city of Blantyre, surrounding townships, and villages. It is also a teaching hospital for different health-related professions.

The Chatinkha maternity unit has seven departments, namely: antenatal clinic, antenatal ward, labor and delivery ward, postnatal ward, neonatal nursery ward, postnatal and family planning clinic, and gynecological ward. Within the labor and delivery ward there is a High Dependency Unit (HDU) where women requiring intensive care from Chatinkha maternity are admitted. Usually clients with severe pre-eclampsia and eclampsia are managed in the HDU, labor ward, and postnatal ward, depending on the severity of their conditions. An average of 25 women with severe pre-eclampsia and 11 with eclampsia are attended to at Chatinkha every month according to 
a QECH health management information system report in $2016 .^{28}$ The backbone of maternity care in the unit are midwives who are either registered Nurse/Midwives or Nurse/Midwife technicians. These midwives provide $\mathrm{MgSO}_{4}$ to clients both intravenously for loading dose and intramuscularly for maintenance dose. The drug comes in different concentrations and the midwives have to dilute to administer the proper concentration and dose to the clients. Additionally, the midwives monitor the progress of the clients regularly. Therefore, the hospital was chosen as the ideal setting for the study because clients on magnesium sulfate were present at the facility.

\section{Sampling and Selection of Study Participants}

We purposively selected 10 midwives for an in-depth interview (IDIs) and seven for a focus group discussion. Purposive sampling enabled the researchers to select study participants who could provide rich information based on their knowledge and exposure ${ }^{24,29}$ to magnesium sulfate administration and monitoring. Variations such as age, years of experience in the maternity unit, educational background, and cadre were considered during the recruitment process. Participants were recruited based on the following criteria; being a midwife regardless of the cadre; having worked in the maternity wing for a period of 3 months and above, exposure to magnesium sulfate administration, and monitoring and willingness to be interviewed pre- and post-intervention.

The researcher and research assistant approached the prospective participants and explained to them the study procedures. Those who met the criteria and were willing to participate were given an information sheet which contained detailed information about the study, and we sought informed consent from them. None of the participants who were approached declined to participate.

\section{Data Collection}

Prior to pre-testing, the interview and discussion guides were reviewed by an independent researcher for content and their feedback was considered in the final guides. We used Zomba Central Hospital, which has similar characteristics with Queen Elizabeth Central Hospital, to pre-test the interview and FGD guides. Following the pre-testing, identified ambiguous areas were corrected accordingly. Participants were informed that the discussions and interviews will be digitally recorded for accuracy and completeness of data, which all participants agreed. Indepth interviews and the FGDs were conducted in one of the rooms in the maternity wing using the pre-tested, semistructured interview guide and discussion guide, respectively, which contained open ended questions with similar information. One broad question guided the interviews and the discussion: "What would you say about the use and monitoring of magnesium sulfate in this unit among midwives?" We probed for more information on: how magnesium sulfate is administered; the availability of policy and guidelines related to magnesium sulfate administration and monitoring; the availability of magnesium sulfate; challenges associated with magnesium sulfate use (administration and monitoring) and how to overcome the challenges throughout the interview period to allow each participant to share their own personal experiences (see Supplementary files 2 and 3). We collected data through IDIs and a FGD to have a broader perspective on magnesium sulfate use as the methods complimented each other. $^{24}$

All IDIs and the FGD were conducted in English and each interview lasted, on average, 35 minutes, while the FGD took 65 minutes. During the FGD the researcher facilitated the discussion while the research assistant who was a midwife with a bachelor's degree took notes on verbal and non-verbal communication which took place during the discussions to enrich the data.

After the discussion and each interview, the main issues presented were written down and summarized to the participants for data verification. Data were triangulated through the data collection methods which were IDIs and FGD to minimize biases. ${ }^{30}$ Data saturation was reached after interviewing eight participants as no new substantive information was gathered, however two more interviews were conducted to validate the data saturation. ${ }^{31}$

\section{Data Management and Analysis}

Soon after data collection, the researchers transcribed the recorded data verbatim. The recorded information, field summaries, and transcripts were kept in a lockable cupboard accessible by the researchers only. The computer with the data had a password known by one of the researchers (MCC). The transcripts were managed in NVivo 10.0. The data were analyzed using the thematic analysis framework, which allows the data to be coded deductively and inductively ${ }^{32}$ with the assistance of CFIR concepts in order to gather information related to 
magnesium sulfate use. The principal investigator (MCC) read all the transcripts against the recorded data and selected important issues in relation to the objective of the study. One transcript was deductively and inductively coded by MCC and was then reviewed by co-investigators and an independent researcher. An agreement was made on the codes to be used for coding the rest of the transcripts while paying attention to emerging codes. Based on the similarities, recurrence, and differences across the data set with the aid of CFIR concepts (Table 1), the codes were organized into categories and themes which were presented as results after the verification process, where MCC checked the themes against the recorded data. We used strategies recommended for enhancing validity such as triangulating source and data collection methods, use of

Table I Development of Themes Using CFIR Concepts

\begin{tabular}{|c|c|c|}
\hline CFIR Domain & $\begin{array}{l}\text { CFIR } \\
\text { Concept }\end{array}$ & Finding \\
\hline $\begin{array}{l}\text { Intervention } \\
\text { characteristic }\end{array}$ & Complexity & $\begin{array}{l}\text { Number of steps are required } \\
\text { to manage pregnant women on } \\
\text { magnesium sulfate, leading to } \\
\text { the practice being } \\
\text { labor intensive }\end{array}$ \\
\hline Outer setting & $\begin{array}{l}\text { Protocols/ } \\
\text { guidelines/ } \\
\text { formula/ } \\
\text { regimen }\end{array}$ & $\begin{array}{l}\text { Guidelines/protocols on } \\
\mathrm{MgSO}_{4} \text { use are vital Although } \\
\text { they exist in some settings, } \\
\text { uniformity is a problem } \\
\mathrm{MgSO}_{4} \text { supplied is in different } \\
\text { formulas, making it difficult to } \\
\text { calculate the required dose }\end{array}$ \\
\hline Inner setting & $\begin{array}{l}\text { Learning } \\
\text { climate }\end{array}$ & $\begin{array}{l}\text { Access to information and } \\
\text { knowledge on management of } \\
\text { clients on } \mathrm{MgSO}_{4} \text { inadequate } \\
\text { Resources for } \mathrm{MgSO}_{4} \text { use such } \\
\text { as training and equipment for } \\
\text { monitoring clients inadequate }\end{array}$ \\
\hline $\begin{array}{l}\text { Individual } \\
\text { characteristics }\end{array}$ & $\begin{array}{l}\text { Knowledge and } \\
\text { belief about } \\
\mathrm{MgSO}_{4}\end{array}$ & $\begin{array}{l}\text { Lack of confidence to } \\
\text { administer } \mathrm{MgSO}_{4} \text { due to poor } \\
\text { knowledge and skills } \\
\text { Perception that } \mathrm{MgSO} 4 \text { has } \\
\text { adverse side-effects, lack of } \\
\text { experience and lack of interest }\end{array}$ \\
\hline $\begin{array}{l}\text { Implementation } \\
\text { process }\end{array}$ & $\begin{array}{l}\text { Engaging and } \\
\text { executing }\end{array}$ & $\begin{array}{l}\text { Provide adequate support to } \\
\text { encourage proper use of } \\
\mathrm{MgSO}_{4} \text { through training } \\
\text { Orient newly qualified } \\
\text { midwives on } \mathrm{MgSO}_{4} \\
\text { Provide supportive supervision }\end{array}$ \\
\hline
\end{tabular}

probes during data collection, member checking after each interview and discussion, prolonged immersion into data, and inclusion of direct quotes in the results section. Prolonged immersion of the researcher (MC) by reading the transcripts several times, piloting of the research instruments, using the same interview and discussion guides, and inclusion of direct quotes in the results section assured credibility and dependability. Conformability was achieved by data triangulation through source and methods as information collected from multiple sources and methods help in confirming emerging issues. Variations which were observed during recruitment procedures facilitated the transferability of the study as views came from individuals with a variety of experience.

\section{Ethical Considerations}

Permission to conduct the study was granted by the College of Medicine Research and Ethics Committee (COMREC) Certificate No P.01/17/2092. Permission was also sought from Queen Elizabeth Central Hospital Management and individual heads of departments where the participants were drawn. All the participants were assured that their participation in the study was voluntary and written informed consent was obtained prior to the interviews and discussions. Voluntary participation and informed consent ensured that our study complied with ethical requirements. Additionally, the researchers ensured the research participants that they could withdraw at any time without facing reprimands. Confidentiality and anonymity of the participants were observed by conducting interviews in private places and using codes for identification of the participants. The participants were informed that the information they provided would be used to improve services.

\section{Results}

\section{Demographic Characteristics of the Participants}

There were 17 participants, of which only one was male. The participants' ages ranged from 24 to 60 years. Work experience of the participants ranged from 1-18 years in a maternity unit. All the participants were nurse/midwife technicians except five, four of whom were registered nurse/midwives with a bachelor's degree and one who was a holder of a master's degree in Midwifery. Five nurse/midwives were from the labor ward, three from the antenatal ward, five from the postnatal ward, two 
Table 2 Demographic Characteristics of the Study Participants $(n=17)$

\begin{tabular}{|l|l|}
\hline Variable & Finding \\
\hline Median age in years & 35 \\
\hline Gender & \\
Male & 1 \\
Female & 16 \\
\hline Work Experience (years of service) & \\
Less than a year & 2 \\
I-5 years & 6 \\
6-I0 years & 4 \\
Above I0 years & 5 \\
\hline Cadre & \\
Registered nurse/midwife & 5 \\
Nurse/midwife technician & 12 \\
\hline Place of Work & \\
Gynecology ward & 2 \\
Antenatal ward & 3 \\
Labor and delivery ward & 5 \\
Post-natal ward & 5 \\
Maternity HDU & 2 \\
\hline
\end{tabular}

from the gynecological ward, and two from maternity HDU (see Table 2).

\section{Emerged Theme}

We identified one overarching theme: "Inadequate governing approaches on management of clients on $\mathrm{MgSO}_{4}$ " with corresponding sub-themes; in adequate preparation on magnesium sulfate administration; inconsistent formula, regimen, guidelines and protocols on magnesium sulfate and lack of resources.

The narratives of the participants indicated that there was a general feeling that magnesium sulfate administration and monitoring was difficult, particularly among those midwives who were not working in the labor ward as per quote below

The problem is the more clients you see the more competent you become .... I have been in the labor ward and I do give magnesium sulfate a lot .... am so competent but I know people from other wards and those without experience struggle to give it and some pretend to be busy to avoid giving it. (Midwife 3, Focus group)

The participants explained the reasons $\mathrm{MgSO}_{4}$ is a difficult drug to administer and monitor under the following subthemes:

\section{Inadequate Preparation on Magnesium} Sulfate Administration and Monitoring

Participants from focus group discussions felt the training they received, particularly in the pre-service, was mostly theory. They had little practical experience, hence limited competence and confidence related to $\mathrm{MgSO}_{4}$ use. Consequently, the participants did not give the drug to the clients.

at school they should focus most on the practical side, you might experience a patient presenting with such condition so when you do, do this ... but also they should not hide that you might be afraid to administer it (Magnesium sulfate). (Midwife 4, Focus group discussion)

Participants felt that some of the reasons for the limited experience that is acquired during pre-service training is the large number of students in classes. This point was raised by one of the participants as follows;

... but due to too many students, you have no hands on experience $\mathrm{mmmh}$, they say you learn a lot with practice. (Midwife 5, FGD)

In addition, participants from the in-depth interview group felt that there is no deliberate effort to reorient newly recruited midwives on $\mathrm{MgSO}_{4}$ use once they join the health system, a practice which does not encourage them to view magnesium sulfate as a drug which they can ably give

... so that time I was using the knowledge I learnt from school it was hectic ... but if those that are just coming from school were oriented on this emergency drug that would help. (Midwife 1, In-depth interview)

I had no orientation at the work place on magnesium sulphfate use and it was difficult. (Midwife 10, In-depth interview)

Participants had beliefs which made them reluctant to use $\mathrm{MgSO}_{4}$ properly: The beliefs were that magnesium sulfate is a dangerous drug which has adverse side-effects and that it should only be administered in special areas.

People are fearful to give especially those from health centers they feel that the ones who can administer it are only nurse/midwives from central hospitals like here at Queen Elizabeth Central hospital. (Midwife 3, FGD)

Participants felt training midwives on magnesium sulfate use at the workplace through refresher courses and supportive supervision would improve magnesium sulfate use among midwives:

from my experience at the postnatal ward. mmmh some are not comfortable administering magnesium sulfate 
especially when they are alone. They are not confident enough to do it on their own, they think they might end up messing the dose so they would wait even when it's an emergency that's why am saying there is need for refresher training courses so that the staff can be reminded. (Midwife 8, In-depth interview)

Another participant added that

there is need to update each other whether there can be a fresher course at ward level. The course can be organized despite the busy schedule of midwives ... in addition, we need matrons to come at our wards so that they can help us with the areas we are having trouble with when it comes to magnesium sulfate use. (Midwife 10, Indepth interview)

Although attempts have been made to improve magnesium sulfate use through in-service training, the participants reported that the training does not target midwives working outside the labor ward. They were of the opinion that in-service training on magnesium sulfate use should be provided to all midwives in the maternity unit frequently in order to save lives of mothers.

I think the trainings should be for all midwives in the maternity unit because we don't know who is going to have an eclamptic patient ... it can be a midwife in the postnatal or it can happen in theater even a midwife on locum can have such an experience so you cannot wait for a labor ward midwife to come to your rescue it's an emergency ... and the trainings should be every now and then, an ongoing event, not annually or every two years. (Midwife 5, In-depth interview)

Participants who were registered midwives felt that administering and monitoring magnesium sulfate was not difficult, only that the midwives were lazy.

aah some are lazy they feel that the drug is demanding so it would mean creating more work, there is a lot of paperwork involved, since you have to document properly so ... sometimes we (midwives) are lazy to document properly. (Midwife 9, In-depth interview)

\section{Inconsistent Formula, Regimen, and Guidelines/Protocols on Magnesium Sulfate Use}

Participants from both the focus group and in-depth interviews expressed that the calculation of magnesium sulfate is hard and this is complicated by the fact that the drug comes in different formulas and in some cases different regimen of magnesium sulfate are prescribed.

The other challenge which I have observed is that the drug comes in different formulas, some come in ampoules in mls yet you are expected to give it in grams so it becomes difficult for other people to prepare such a drug and give it to a patient. (Midwife 2, In-depth interview)

... Because you go to a district hospital, they will say, the right dosage is the same 4 grams you put in $250 \mathrm{~mL}$ of normal saline. When you come here at Queens they will tell you to add $12 \mathrm{~mL}$ of water to make $20 \mathrm{~mL}$ and you give a slow IV push ... so it looks like different hospitals have different protocols.

In some cases midwives reported that guidelines/protocols on magnesium sulfate administration and monitoring were not available in their units, which complicate implementation of the intervention.

\section{Lack of Resources}

Participants felt that administration and monitoring of clients on magnesium sulfate is labor intensive as one has to carry out observations at specific times and it requires proper documentation. Therefore it cannot work in the facilities due to the shortage of midwives and equipment.

it is difficult to give and monitor when there are few midwives on duty ... you cannot carry out the observations properly. (Midwife 6, FGD).

to give the drug you have to monitor the client before and after giving the drug frequently ... eeh lets say every hour or $4 \mathrm{hr}$ and document not easy! (Midwife3, In-depth interview)

Another participant added and narrated as follows;

Lack of resources is also another challenge, ie, bp machine as a result you cannot monitor the clients. (Midwife 8, Indepth interview)

However, one discussant explained that some omissions occur in carrying out other observations due toa lack of knowledge and skill on some observations and the feeling that other observations are to be done by doctors

midwives most of the time just do it without particular skill ("we just hit there") and sometimes we don't even do it, we prioritize the urine output and respiratory observations, things we are comfortable of doing (Midwife 4, FGD) 
Another participant narrated that;

What is difficult with mag sulfit's the calculations, maybe you wrongly administer a wrong dose and you give it through the wrong route.

Although participants acknowledged that guidelines and protocols on magnesium sulfate use can act as a useful tool to encourage the practice of magnesium sulfdate use among midwives, they felt that the guidelines are not uniform. Each facility has got its own guidelines which brings about confusion.

\section{Discussion}

Our findings have demonstrated that there was suboptimal use of magnesium sulfate in the maternity wing as an intervention, particularly among midwives working in other units apart from the labor ward. Contributing factors to improper use of magnesium sulfate emanated from the intervention itself, the individual, the setting, and the process by which magnesium sulfate administration and monitoring is implemented. Participants felt that the intervention of administering and monitoring magnesium sulfate is demanding and requires appropriate knowledge, attitude, and skills. The perceived lack of knowledge, skill, resources, and uniform guidelines on the drug administration and monitoring were some of the factors highlighted among the midwives.

In our study, at individual level midwives felt that they did not acquire the expected knowledge, attitude, and skills related to $\mathrm{MgSO}_{4}$ use in either in-service or preservice training.

Our finding on the inadequate training as a factor contributing to poor magnesium sulfate use is similar to what other studies have reported, ${ }^{4,13,19}$ despite studies showing that midwives receive more intensive training and clinical updates in the pre-service on $\mathrm{MgSO}_{4}$ use as compared to obstetricians. $^{4}$

In our study, lack of skill in conducting knee jerk was perceived as a factor affecting $\mathrm{MgSO}_{4}$ monitoring, yet loss of the knee jerk is the first warning sign of magnesium sulfate toxicity. ${ }^{1,2}$ Additionally individual beliefs that $\mathrm{MgSO}_{4}$ is a toxic drug which can lead to adverse sideeffects discouraged midwives to administer $\mathrm{MgSO}_{4}$, as reported in other studies. ${ }^{1}$ These results necessitate knowledge and skill enhancement among midwives which will assist them to administer and monitor the drug with confidence and comfort timely, ${ }^{13}$ hence development of a positive attitude among midwives towards $\mathrm{MgSO}_{4}$ use.
Although knowledge and skill attainment is not a guarantee for behavior change, knowledge is a prerequisite for best practices. ${ }^{33}$ To sustain the knowledge and skills there is a need to provide the in-service education frequently and provision of supportive supervision. Similar to our studies, other studies have also advocated for supportive supervision and periodic trainings to improve $\mathrm{MgSO}_{4} \cdot{ }^{4,34}$ Furthermore, there is a need to reorient new midwives and provide them with mentors. In our study participants reported that trainings are only provided to midwives working in the labor ward, a finding which defeats the purpose of $\mathrm{MgSO}_{4}$ use as a lifesaving drug at any point during the perinatal period. We therefore propose that all midwives should receive frequent training on magnesium sulfate use. During the training, emphasis should be made that magnesium sulfate is a safe drug using the existing evidence to reduce the fears that magnesium sulfate is a toxic drug. Furthermore, midwives should be encouraged to carry out observations on clients who have received magnesium sulfate, as expected to generate evidence on side-effects of $\mathrm{MgSO}_{4}$. Such information may also assist in expelling the belief that magnesium sulfate is a dangerous drug. Furthermore, deliberate efforts should be made for midwives not working in the labor ward to have drills and to work in the labor ward on clients with severe pre-eclampsia and eclampsia as part of their practicals for their Continuous Professional Development, which currently is only focusing on theory.

Factors affecting administration and monitoring of clients on magnesium sulfate were also related to setting and the process of the implementation of magnesium sulfate use.

Our results have shown that guidelines on magnesium sulfate administration and monitoring, though useful, were not available in other units, making implementation of magnesium sulfate administration and monitoring difficult for other midwives. In addition, midwives expressed that $\mathrm{MgSO}_{4}$ was of different formulas and different regimens were prescribed for eclamptic patients or patients with severe preeclampsia, which makes administration of $\mathrm{MgSO}_{4}$ a hard process. We propose reorienting of both new and old midwives on $\mathrm{MgSO}_{4}$ guidelines, protocols, different formulas, and regimen be an ongoing activity. The reorientation will assist in instilling confidence, improving self-efficacy and readiness to administer magnesium sulfate among midwives, and ultimately improving the process of $\mathrm{MgSO}_{4}$ use. 
In agreement with results from other studies, ${ }^{18,35}$ both human and material resources were viewed as deterrents for magnesium proper. However in our study, material resources reported include a blood pressure monitoring machine, while Katageri et al's ${ }^{13}$ study reported the absence of protein dip sticks as a factor barring $\mathrm{MgSO}_{4}$ use. The reason for not mentioning protein dipsticks in this study may indicate the dip sticks were available or it was not part of the assessment midwives conducted to monitor progress of clients on $\mathrm{MgSO}_{4}$ use. In addition, our study did not identify magnesium sulfate stock outs as a factor contributing to improper use of $\mathrm{MgSO}_{4}$ , a finding which has been reported in the other studies. ${ }^{35,37}$

\section{Conclusion}

Midwives perceived $\mathrm{MgSO}_{4}$ use as a demanding activity due to inadequate training, inconsistent tools, and lack of resources. While periodic in-service training should be intensified to improve $\mathrm{MgSO}_{4}$ use, necessary resources should be provided, such as blood pressure machines, more midwives, and protocols.

\section{Study Strengths and Limitations}

We consider the use of participants working in the maternity wing with different levels of education and experience as a strength, since information generated came from different perspectives. Additionally, using different methods for data collection enriched the data further as the methods complemented each other. The study had limitations as follows: First, only one health facility was used and the sample size was small, as such the findings cannot be generalized. Second, a possible weakness in this study could be participants giving desirable responses to the facilitator, whom they knew was a midwife. However, this was minimized by techniques such as probing and member checking, which were employed to ensure that the responses were reliable.

\section{Abbreviations}

COMREC, College of Medicine Research Ethics Committee; FGD, Focus group discussion; IDIs, In depth interviews; LMICs, Low and middle income countries; $\mathrm{MgSO}_{4}$, Magnesium sulfate; NORHED; The Norwegian Programme for Capacity Development in Higher Education and Research for Development; QECH, Queen Elizabeth Central Hospital; WHO, World Health Organization.

\section{Ethics Approval and Consent to Participate}

Permission to conduct the study was granted by the College of Medicine Research Ethics Committee (COMREC) certificate number P.01/17/2092, while institutional permission was obtained from Queen Elizabeth Central Hospital management and from the head of Chatinkha maternity wing. All the participants were 18 years old and above and their participation in the study was voluntary. Informed consent was obtained prior to the interviews and discussions. Voluntary participation and informed consent ensured that our study complied with ethical requirements. Additionally, the researchers ensured the research participants that they could withdrawal at any time without facing reprimands. Confidentiality and anonymity of the participants were observed by conducting interviews in private places and using codes for identification of the participants. The participants were informed that the information they provided would be used for quality improvement and could be published.

\section{Data Sharing Statement}

All datasets used or analyzed during the current study are available from the corresponding author on reasonable request.

\section{Acknowledgments}

We would like to thank the participants for their time and willingness to share their views.

\section{Author Contributions}

All authors contributed to data analysis, drafting or revising the article, gave final approval of the version, and agreed to be accountable for all aspects of the work.

\section{Funding}

This research was funded by NORHED Project MWI- 13/ 0032 at KCN under competitive research grant scheme for faculty.

\section{Disclosure}

The authors declare that they have no competing interests in this work.

\section{References}

1. Smith JM, Lowe RF, Fullerton J, Currie SM, Harris L, Felker-Kantor E. An integrative review of the side effects related to the use of magnesium sulfate for pre-eclampsia and eclampsia management. BMC Pregnancy Childbirth. 2013;13:34. doi:10.1186/1471-2393-13-34 
2. Tukur J. The use of magnesium sulphate for the treatment of severe pre-eclampsia and eclampsia. Ann Afr Med. 2009;8(2):76. doi:10.4103/1596-3519.56232

3. Smith JM, Currie S, Cannon T, Armbruster D, Perri J. Are national policies and programs for prevention and management of postpartum hemorrhage and preeclampsia adequate? A key informant survey in 37 countries. Glob Health. 2014;2(3):275-284.

4. Long Q, Oladapo OT, Leathersich S, et al. Clinical practice patterns on the use of magnesium sulphate for treatment of pre-eclampsia and eclampsia: a multi-country survey. BJOG. 2017;124(12):1883-1890. doi:10.1111/1471-0528.14400

5. Magpie Trial Follow-Up Study Collaborative. The Magpie Trial: a randomised trial comparing magnesium sulphate with placebo for pre-eclampsia. Outcome for women at 2 years. BJOG. 2007;114 (3):300-309. doi:10.1111/j.1471-0528.2006.01166.x

6. Altman D, Carroli G, Duley L, et al.; Magpie Trial Collaboration Group. Do women with pre-eclampsia, and their babies, benefit from magnesium sulphate? The Magpie Trial: a randomised placebo-controlled trial. Lancet. 2002;359(9321):1877-1890.

7. Vousden N, Lawley E, Seed PT, et al. Incidence of eclampsia and related complications across 10 low-and middle-resource geographical regions: secondary analysis of a cluster randomised controlled trial. PLoS Med. 2019;16(3):e1002775. doi:10.1371/journal. pmed.1002775

8. Alkema L, Chou D, Hogan D, et al. Global, regional, and national levels and trends in maternal mortality between 1990 and 2015, with scenario-based projections to 2030: a systematic analysis by the UN Maternal Mortality Estimation Inter-Agency Group. Lancet. 2016;387(10017):462-474. doi:10.1016/S0140-6736(15)00838-7

9. Mataya RM. 2012.Republic of Malawi Ministry of Health Report on the Confidential Enquiry into Maternal Deaths in Malawi (20082012). Available from: http://mdsr-action.net/wp-content/uploads/ 2016/04/MOH_2016_Final-Malawi-CEMD-Report-18-09-14.pdf. Accessed July 10, 2018.

10. Lema VM, Changole J, Kanyighe C, Malunga E. Maternal mortality at the queen Elizabeth central teaching hospital, Blantyre, Malawi. East Afr Med J. 2005;82(1). doi:10.4314/eamj.v82i1.9286

11. UNICEF. Maternal and Newborn Health Disparities in Malawi; 2016.

12. Browne JL, Van Nievelt SW, Srofenyoh EK, Grobbee DE, KlipsteinGrobusch K. Criteria-based audit of quality of care to women with severe pre-eclampsia and eclampsia in a referral hospital in Accra, Ghana. PLoS One. 2015;10(4):e0125749.

13. Katageri G, Charantimath U, Joshi A, et al. Availability and use of magnesium sulphate at health care facilities in two selected districts of North Karnataka, India. Reprod Health. 2018;15(1):91. doi:10.1186/s12978-018-0531-6

14. De Silva DA, Proctor L, von Dadelszen P, McCoach M, Lee T, Magee LA. Determinants of magnesium sulphate use in women hospitalized at $<29$ weeks with severe or non-severe pre-eclampsia. PLoS One. 2017;12(12):e0189966.

15. WHO. WHO Recommendations for Prevention and Treatment of PreEclampsia and Eclampsia. Vol. 2015. Geneva: WHO; 2011.

16. Nagaria T, Mitra S, Banjare SP. Single Loading Low Dose MgSo(4) Regimen: a Simple, Safe and Effective Alternative to Pritchard's Regimen for Indian Women. J Clin Diagn Res. 2017;11(8):QC08QC12. doi:10.7860/JCDR/2017/26635.10453

17. Mahajan NN, Thomas A, Soni RN, Gaikwad NL, Jain SM. 'Padhar Regime'-a low-dose magnesium sulphate treatment for eclampsia. Gynecol Obstet Invest. 2009;67(1):20-24. doi:10.1159/000158647

18. Oguntunde O, Charyeva Z, Cannon M, et al. Factors influencing the use of magnesium sulphate in pre-eclampsia/eclampsia management in health facilities in Northern Nigeria: a mixed methods study. $B M C$ Pregnancy Childbirth. 2015;15(1):130. doi:10.1186/s12884-0150554-8
19. Sheikh S, Qureshi RN, Khowaja AR, et al. Health care provider knowledge and routine management of pre-eclampsia in Pakistan. Reprod Health. 2016;13(2):104. doi:10.1186/s12978-016-0215-z

20. Sheth SS, Chalmers L. Magnesium for preventing and treating eclampsia: time for international action. Lancet. 2002;359 (9321):1872-1873. doi:10.1016/S0140-6736(02)08783-4

21. Damschroder LJ, Aron DC, Keith RE, Kirsh SR, Alexander JA, Lowery JC. Fostering implementation of health services research findings into practice: a consolidated framework for advancing implementation science. Implement Sci. 2009;4(1):50. doi:10.1186/17485908-4-50

22. Kirk MA, Kelley C, Yankey N, Birken SA, Abadie B, Damschroder L. A systematic review of the use of the consolidated framework for implementation research. Implement Sci. 2015;11 (1):72. doi:10.1186/s13012-016-0437-z

23. Keith RE, Crosson JC, O'Malley AS, Cromp D, Taylor EF. Using the Consolidated Framework for Implementation Research (CFIR) to produce actionable findings: a rapid-cycle evaluation approach to improving implementation. Implement Sci. 2017;12(1):15. doi:10. 1186/s13012-017-0550-7

24. Polit DF, Beck CT. Essentials of Nursing Research: Appraising Evidence for Nursing Practice. Lippincott Williams \& Wilkins; 2013.

25. Creswell JW, Klassen AC, Plano Clark VL, Smith KC. Best Practices for Mixed Methods Research in the Health Sciences. Vol. 2013. Bethesda (Maryland): National Institutes of Health; 2011:541-545.

26. Vaismoradi M, Turunen H, Bondas T. Content analysis and thematic analysis: implications for conducting a qualitative descriptive study. Nurs Health Sci. 2013;15(3):398-405. doi:10.1111/nhs.2013.15. issue-3

27. Venkateshi V, Brown SV, Bala H. Bridging the qualitative-quantitative divide: guidelinesfor conducting mixed methods research in information systems. MIS Q. 2013;1-20.

28. Queen Elizabeth Central Hospital. Health Management Information System; 2016.

29. JW C. Research Design. International Student Edition: Qualitative, Quantitative and Mixed Methods Approaches. 4th ed. India: Sage; 2014.

30. Grove SK, Gray JR. Understanding Nursing Research: Building an Evidence-Based Practice. Elsevier Health Sciences; 2014.

31. Boyatzis RE. Transforming Qualitative Information: Thematic Analysis and Code Development. Thousand Oaks: sage; 1998.

32. Braun V, Clarke V. Using thematic analysis in psychology. Qual Res Psychol. 2006;3(2):77-101. doi:10.1191/1478088706qp063oa

33. Glanz K, Rimer BK, Viswanath K. Health Behavior and Health Education: Theory, Research, and Practice. John Wiley \& Sons; 2008.

34. Babu G, Singh V, Nandy S, Jana S, TN S, SM S. Supportive supervision and immunization coverage: evidence from India. Internet J Epidemiol. 2011;9.

35. Lotufo FA, Parpinelli MA, Osis MJ, Surita FG, Costa ML, Cecatti JG. Obstetrician's risk perception on the prescription of magnesium sulfate in severe preeclampsia and eclampsia: a qualitative study in Brazil. PLoS One. 2017;12(3):e0172602. doi:10.1371/journal.pone.0172602

36. Barua A, Mundle S, Bracken H, Easterling T, Winikoff B. Facility and personnel factors influencing magnesium sulfate use for eclampsia and pre-eclampsia in 3 Indian hospitals. Int $J$ Gynecol Obstet. 2011;115(3):231-234. doi:10.1016/j.ijgo.2011.07.016

37. Ridge AL, Bero LA, Hill SR. Identifying barriers to the availability and use of magnesium sulphate injection in resource poor countries: a case study in Zambia. BMC Health Serv Res. 2010;10(1):340. doi:10.1186/1472-6963-10-340 


\section{Publish your work in this journal}

The International Journal of Women's Health is an international, peerreviewed open-access journal publishing original research, reports, editorials, reviews and commentaries on all aspects of women's healthcare including gynecology, obstetrics, and breast cancer. The manuscript management system is completely online and includes a very quick and fair peer-review system, which is all easy to use. Visit http://www.dovepress.com/testimonials.php to read real quotes from published authors.

Submit your manuscript here: https://www.dovepress.com/international-journal-of-womens-health-journal 